\title{
An Accurate and Compact High Power Monocycle Pulse Transmitter for Microwave Ultra-Wideband Radar Sensors with an enhanced SRD model: Applications for Distance Measurement for lossy Materiel
}

\author{
Younes AHAJJAM ${ }^{1,3}$, Otman AGHZOUT $^{1,2}$, Josè M. CATALA-CIVERA ${ }^{3}$, \\ Felipe PEÑARANDA-FOIX ${ }^{3}$, and Abdellah DRIOUACH ${ }^{1}$ \\ ${ }^{1}$ Dept. of Physics, Faculty of Science, Abdelmalek Essaadi University, Tetouan, Morocco. \\ ${ }^{2}$ Department of Telecommunication, ENSA. LabSIT-FS, UAE Univertisy, Tetouan, Morocco \\ ${ }^{3}$ Instituto ITACA. Universitat Politècnica de València. Camino de Vera s/n. 46022 Valencia, España. \\ *corresponding author, E-mail: ahajjam. younes egmail.com
}

\begin{abstract}
In This paper, a high power sub-nanosecond pulse transmitter for Ultra-wideband radar sensor is presented. The backbone of the generator is considered as a step recovery diode and unique pulse injected into the circuit, which gives rise to an ultra-wideband Gaussian pulse. The transistor driver and transmission line pulse forming the whole network are investigated in detail. The main purpose of this work is to transform a square waveform signal to a driving pulse with the timing and the amplitude parameters required by the $S R D$ to form an output Gaussian pulse and then into high monocycle pulses. In simulation aspect, an improved output response is required, in this way a new model of step recovery diode has been proposed as a sharpener circuit. This proposition was applied to increase the rise-time of the pulses. For a good range radar, a high amplitude pulse is indispensable, especially when it comes to penetrating the thick lossy material. In order to overcome this challenge, a simple technique and useful solution are introduced to increase the output amplitude of the transmitter. This technique consists to connect the outputs of two identical pulse generators in parallel respecting the restrictions required. The pulse transmitter circuit is completely fabricated using micro-strip structure technology characteristics. Waveforms of the generated monocycle pulses over $10 \mathrm{~V}$ in amplitude with $3.5 \%$ in overshoot have been obtained. Good agreement has been achieved between measurement and simulation results.
\end{abstract}

\section{Introduction}

Recently, and according to Ultra-wideband technology development, increased demands on high-resolution positioning in the lossy material is required. Furthermore, attracted attention by many researchers have been concentrated more and more on sub-nanosecond pulses $[1,3]$; because Ultra-short pulses with well-defined output waveform plays a crucial role in the $U W B$ transmitter design. A subnanosecond pulse transmitter is the fundamental part of any ultra-wideband $(U W B)$ radar sensor [4]. Because the wide bandwidth of UWB pulses ensures fine range resolution and high penetration capability. Instead of higher frequencies, which the losses are enormous, Lower frequencies up to $3.5 \mathrm{GHz}$ are preferred for the application radar in lossy material. The corresponding pulse width of the generated baseband pulses is approximately 200 ps. There are various techniques to generate UWB pulses around this range. The basis of a classical $U W B$ short pulse generator is a driver, a pulse sharpener and a pulse forming network. The driver converts the step-like waveform in Gaussian pulses, and the pulse sharpener converts slow rise time waveform edge to a faster one. However the pulse forming network forms the output pulse to the adequate and favorite pattern: Gaussian or monocycle. Special semiconductor devices are usually utilized as pulse sharpener: avalanche transistor, bipolar diode [5], tunnel diode [6]. , Step Recovery Diode $S R D$ [7], and FETs [8]. Although the disadvantage presented in term of low value of $P R F$, the avalanche diode are very advantageous as high power sharpener due to low power dissipation in the transistor. Tunnel diodes offers the fastest transition time (sub-picosecond) at very low power (about $\mathrm{mW}$ ). The $S R D s$ are a compromise alternative for these devices and offer ultra-short transition time (around $100 \mathrm{ps}$ ) at moderate power levels (changing from hundreds of $\mathrm{mW}$ to tens watt) and with high repetition frequency $[9,10]$. This makes them very appropriate to be used in current $U W B$ generator. Usually, an $S R D$ works as a charge controlled switch, which can alter from low impedance to high impedance state much quickly. This qualification of the $S R D$ is used to sharpen the slow waveform edges. The more details of theory and application of $S R D s$ as pulse sharpening is well described in [11]. The basis of the generated circuit Gaussian pulse generator consists of a simple transistor driver and an $S R D$ sharpener with a pulse-forming circuit. Now a day, it have not an accuracy model of $S R D$ in the simulators software, so a new model of $S R D$ has been inserted in AWR Microwave office and Multisim. Then the simulation accuracy has been improved and the rise-time has been decreased from $630 \mathrm{~ns}$ to $450 \mathrm{~ns}$. The main goal in this paper is to generate high amplitude pulses capable to penetrate thing and lossy materials. To fulfill this task, the outputs of two identical pulse 
generators were connected in parallel. However, a bad connection generates some distortion and ringing in the final output waveform, and then an additional ringing suppression technique are necessary. For this reason an accurate and complete assembling of two identical generator units on a single board with parallel outputs connected has been implemented using a mini-circuit power splitter ZN2PD2. Results shows that, the output of the transmitter power has been increased without any additional effect of ringing, and the peak power of summed pulses is nearly twice of the peak power of the single one. Moreover, adding a Monocycle Forming Network $M F N$, gives a monocycle pulses with $9.7 \mathrm{~V}$ in amplitude and $600 \mathrm{ps}$ in total width. The prototype circuit model has been manufactured using microstrip line technology. All results proves the high performance of the system.

\section{Circuit description and Design for a single impulse generator}

The main parts of the Gaussian pulse generator are a driver and a $S R D$ pulser. The edge-triggered driver generates a well-defined pulse with sufficient power and speed to drive an $S R D$. This pulse is independent of the input waveform amplitude and duty-cycle. In order to minimize the current consumption of the circuit, the input pulse width is set to a few nanoseconds e,g. around 5 ns. The following stage of the pulse generator is the $S R D$ pulser which consists of two main parts. The purpose of an $S R D$ pulse sharpener is to sharpen the leading falling edge of the driving waveform. The sharpened step-like pulse is then processed in a pulseforming circuit to produce a Gaussian-like pulse.

When no input driving pulse is present, the $S R D$ is forward biased by an adjustable constant current source $\mathrm{Ib}$, and a large amount of charge is injected into the diode making the impedance low.

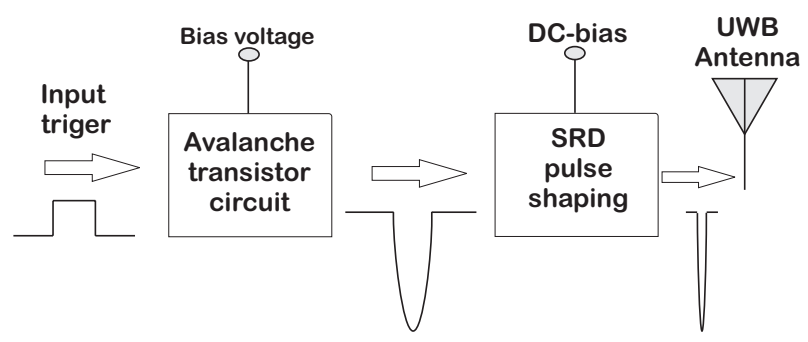

Figure 1: The circuit schematic of the pulse generator.

The circuit schematic of the pulse generator is shown in Fig 1. It is compound for two parts, the avalanche transistor circuit, and $S R D$ pulse sharpener circuit. The pulse generator has been designed and simulated by the AWRMicrowaveof fice and Multisimtransient simulator respectively. The corresponding detailed circuit diagram of the system is shown in Fig.2.

The first part of the generator it's called "Avalanche transistor circuit", which consists of $Q, C_{1}, R_{1}, C_{2}$ and $R_{2}$ as shown in Fig 2. The transistor $Q$ operates as a common-

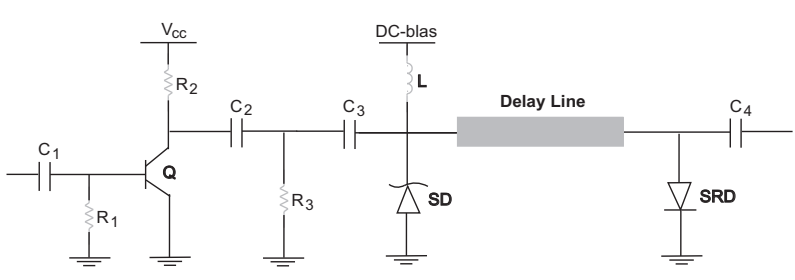

Figure 2: Equivalent circuit of the system.

emitter switch and generates a negatives voltage pulse. The driving waveform passes via a coupling capacitor $C_{2}$ to the $S R D$ pulser. As can be shown in Fig. 2. The discharge current of the avalanche transistor circuit flows from the capacitor $C_{2}$ trough the avalanche transistor and resistor $R_{3}$. The photo of the prototype circuit of the avalanche transistor is shown in Fig.3.

A coupling capacitor $C_{3}$ is added to prohibit the flux of the $D C$ bias current to attain the avalanche transistor circuit. Good responses with $C_{3}$ have been achieved with some $p f$.

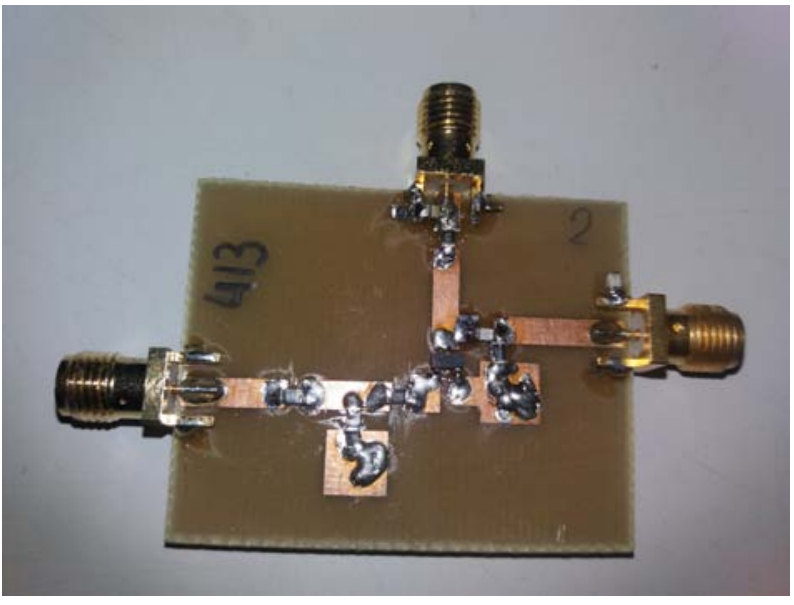

Figure 3: Photo of avalanche transistor circuit.

The second part of the generator it's titled " $S R D$ pulse sharping" which consists of an $S R D$ sharpener and a Gaussian pulse forming the network. The $S R D$, connected in parallel with a transition line, operates as falling edge sharpener.

A photo of the manufactured circuit of the $S R D$ pulse sharpener is shown in Fig.4. The sharpener pulses are processed in a pulse-forming-network circuit, which consists of Schottky diodes and delay lines to produces a Gaussianlike pulse. During the steady-state e.g., forward bias condition, a large amount of charge is injected into the diode, the device appears as a low impedance. After applying the negative driving pulse e.g., reverse biasing condition, the diode continuous as low impedance until all charge is totally removed, at the point where the diode rapidly switches from the low to high impedance. This ability of the $S R D$ to store charge and change its impedance level rapidly is used to sharpen the slow waveform edges. The pulse generator was implemented on an ARLON AD450 substrate of 0.7262 


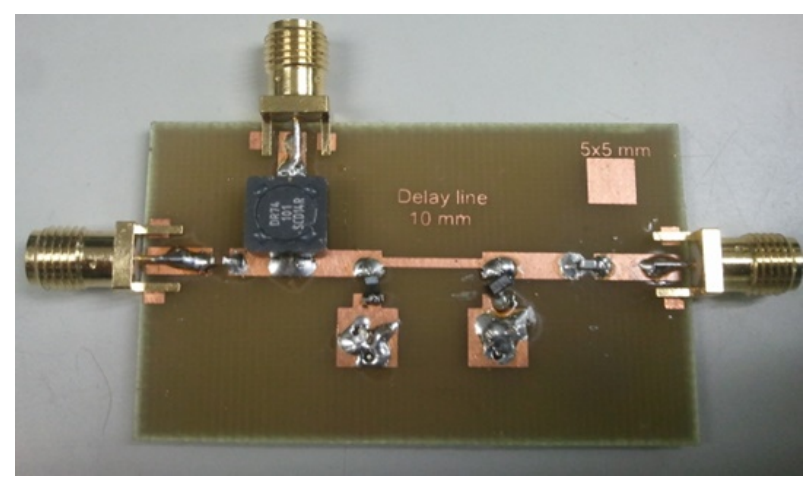

Figure 4: SRD pulse sharping circuit.

$\mathrm{mm}$ in thickness. The waveform was measured using an Agilent $86100 \mathrm{C}$ sampling Oscilloscope at $50 \Omega$ load. For more details about the designed circuit, more descriptions can be found in $[13,14]$. Fig.5 shows the operating mode of $S R D$ pulse sharpening circuit, Which in a steady state, the Schottky diode has a low impedance. When a driving waveform applied to $S R D$ pulse sharpening. The device switches rapidly from a low to high impedance. At this moment, a fast fall-time step waveform propagates in two different directions through the circuit. The first step propagates forward the output, whereas the second one propagates throughout the delay line back to the input. Before the driving waveform was applied, the Schottky diode $(S D)$ was reverse-biased and did not affect the circuit; nevertheless, this diode is now opened by the negative driving pulse and act enough as low impedance to really shortcircuit the transmission line. The step waveform that has been propagating forward the input is reflected back by the low impedance state effect of the diode. Finally, the Gaussian pulse is composed of both step waveforms produced on the circuit; e.g. the step waveform unaltered continue to propagate to the output with the retarded and inverted step waveform.

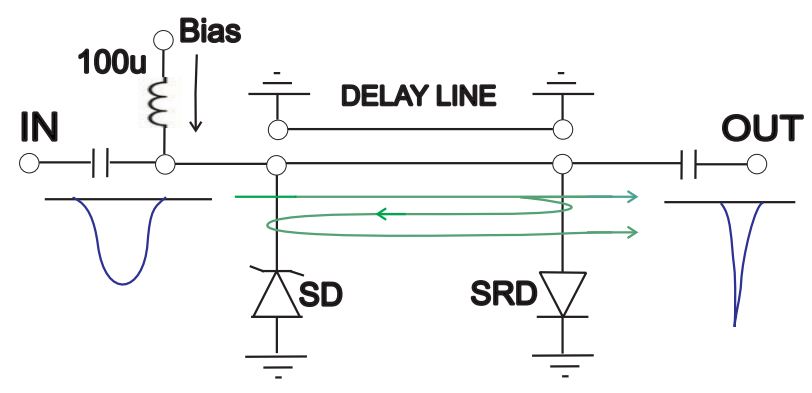

Figure 5: operating mode of SRD pulse sharping circuit.

The $S R D s$ has a crucial role in our generator circuits; ever since the computer simulation of an $S R D$ is a challenging question. This challenge consists significantly of the difference between the $S R D$ and the classical $P N$ junction. In addition, the simulation of the diode models on the Spice simulator provides always incorrect results. Frequently, in conventional time domain Simulators like AWR Microwave office and Multisim Simulators, advanced $S R D$ models are not yet implemented. To face the challenge described above, a new model of $S R D$ has been proposed. The choice of this model was decided after studying many models existing in the literature. One of the advantages of this model is to increase the rise-time. The Fig.6 show the elected voltage-switch model of $S R D$ and its corresponding equivalent circuit. Where $R_{s}$ and $C_{j}$ are the nonlinear impedance capacitance respectively, while $L_{s}$ and $C_{p}$ represent the parasitic parameters of the $S R D$ component.

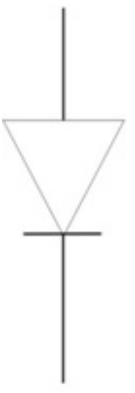

(a)

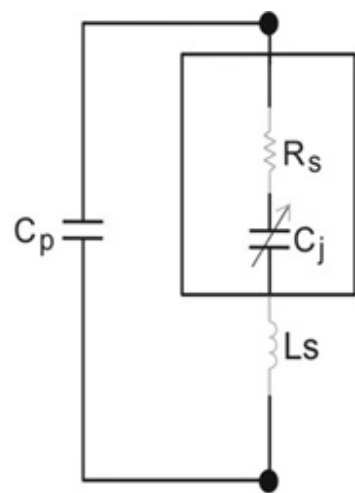

(b)
Figure 6: (a) SRD of AWR Microwave Office. (b) New model of a step recovery diode (SRD).

After many measurements, we have concluded that the diode $M A 44769-287 T$ of $M / A-C O M$ Corporation was the nearest one to our requirements in term of output waveform performance.

Then a basic $S R D$ model proportionate by $A W R$ simulator has been implemented with the parameters adopted from the datasheet of the diode $M A 44769-287 T$. The simulations were realized with the HSPICE transient simulator included in the $A W R$ Microwave Office design suite. We have noted that the elected Diode has a reverse capacitance of $1 \mathrm{pF}$, a minority-carrier lifetime of $10 \mathrm{~ns}$, a minimum transition time of $150 \mathrm{ps}$, a reverse breakdown voltage of $30 \mathrm{~V}$, forward bias current $50 \mathrm{~mA}$ and a $S O D 323$ package. The behavior of the $M A 44769-287 T$ diode is shown in fig.7.

The simulation results are shown in Fig.8. (A) corresponding the output waveform with the usual $S R D$ proposed by the AWR Microwave Office design suite. While the output waveform in Fig. (B) has been generated when the $S R D$ model described below was applied.

The $S R D$ in both results was connected at the input of the microstrip with a $10 \mathrm{~mm}$ in the delay line and $90 \Omega$ a characteristic impedance. The connection of the $S R D$ at the length of the delay line was chosen with the aim to get the highest possible output amplitude. The generated Gaussian pulses are $-6 \mathrm{~V}$ in amplitude and approx. 180psFW HM (Full-Width at Half Maximum) in width. It 


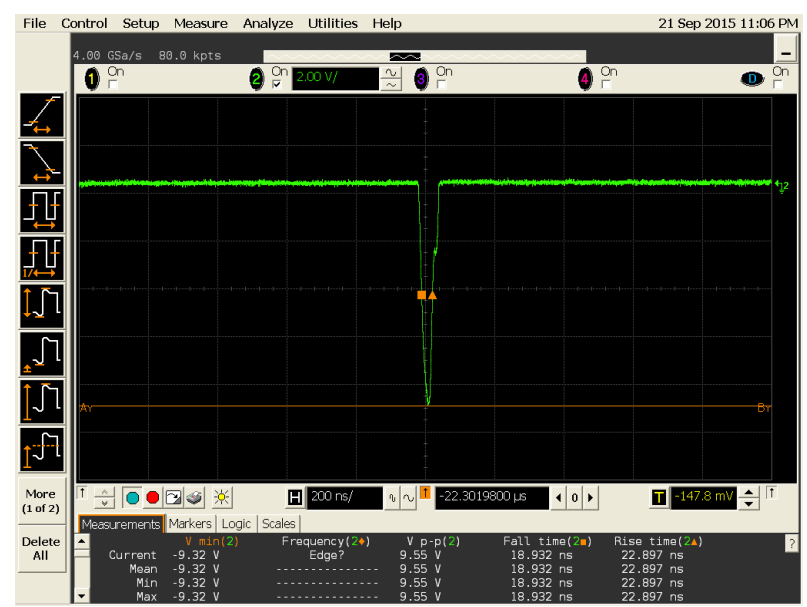

Figure 7: output waveform Gaussian measured using SRD from MA44769-287T
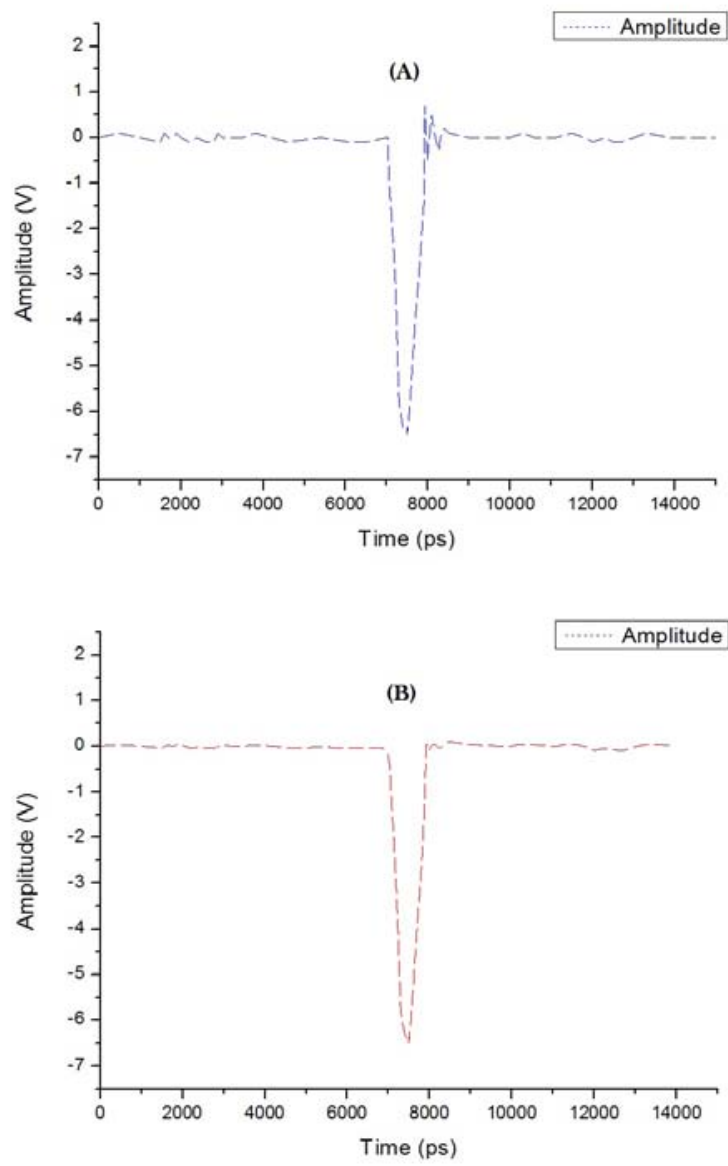

Figure 8: Output waveform before (A) and after (B) the application of new model of SRD respectivily.

is clear to see in the Fig.8. the great difference between the output waveform of the circuit before and after inserting the new model of $S R D$. This differences consisting in remove the most ringing happen just after the descent of the pulse; that leads us to more understanding the great role played by the $S R D$ diode in the formation final of the Gaussian pulse.
The generated Gaussian pulses are now $-6 \mathrm{~V}$ in amplitude with $3.5 \%$ in overshoot.

\section{Powerful Monocycle pulse transmitter design}

For long-range detection or in measurement when penetrating thick lossy obstacles, the high amplitude pulses are much desired. However, high power $U W B$ pulse generators are expensive and are in most cases not compatible with the modern planar technology. To overcome this challenge, there are many ways to increase the output power. One of them is to combine the output waveforms from two or more sources. But combining ultra-wideband Gaussian pulses is a difficult task. The conventional power combining structures, e.g. Wilkinson power divider, are basically narrowband and distort $U W B$ waveforms; In addition, applying a short $U W B$ pulse results in unwished reflections and ringing at other ports. Some dividers/combiners using planar multilayer techniques have also been described in the literature $[15,8]$. Regrettably those dividers/combiners show proper $U W B$ performance, but unfortunately are suitable primarily for the higher $U W B$ band ranging between (3.1-10.6) GHz. The goal of our design is to obtain a powerful waveform with a minimum insertion loss. This requirement is well achieved using a Mini-circuit called power splitter $Z N 2 P D 2$ which works in the band (0.5-5) GHz. An equivalent circuit of this $Z N 2 P D 2$ power splitter was designed in Dimas laboratory at ITACA institute in University Polytechnic of Valencia. It will be published in the few next months.

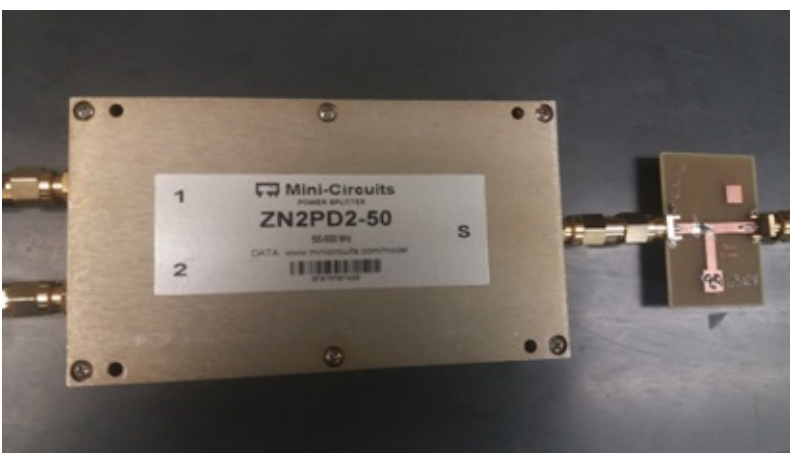

Figure 9: Photo of the Mini-circuit power splitter ZN2PD2.

A block diagram of the proposed powerfull monocycle pulse transmitter is shown in Fig.10. The transmitter consists of two $S R D$ pulse sharping circuit connnected in parallel after the avalanche transistor circuits. Both sharpening circuit are synchronized and trigged by one timing source. The outputs of two identical pulse generators are combined in order to obtain higher output pulse in amplitude.

The monocycle pulses are a special interest because their spectrum does not contain low-frequency components and the implementation of MFN is an easy task. For this 


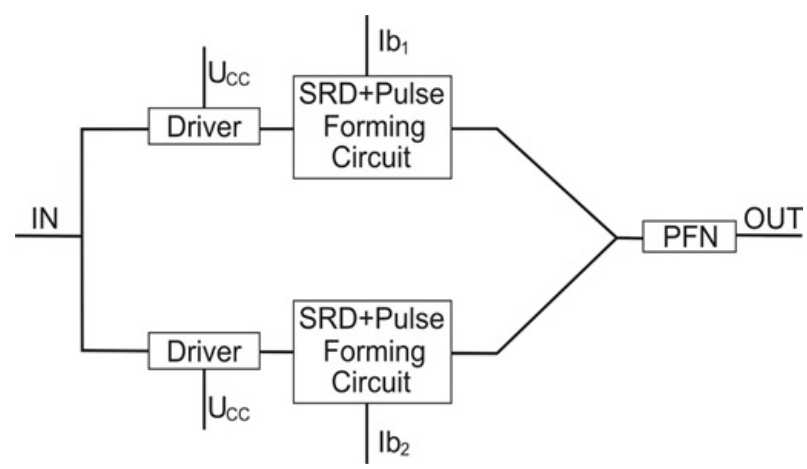

Figure 10: Block diagram of the proposed Radar pulseS transmitter.

reason, the Monocycle Forming Network $(M F N)$ inspired by [16] was connected in the output of the transmitter in order to form the monocycle pulses. The photo of the fabricated circuit and layout are shown in Fig. 11. It consists of a shortened stub with $10 \mathrm{~mm}$ in length and a Schottky diode (BAT15), which reduces significantly the output ringing. The impedance of all micro-strip lines was fixed at $50 \Omega$.

The photo of the proposed powerful compound monocycle pulse transmitter is shown in Fig.12. It is made up of five parts, an avalanche transistor circuit, the splitter divider $E C @ D V P$ which divide the Gaussian waveform generated by the transistor avalanche into identical one, Two $S R D$ pulse sharpening circuit connected in parallel, The power splitter $Z N 2 P D 2$, and an $M F N$.

To test all functionality of the whole system, we collected two identical pulse generators on a single board. In order to obtain the sum of the pulses without any disturbance, it's important that both generators provide an output pulse exactly at the same time. The pulse amplitude is now over $10 \mathrm{~V}$ compared with $4.8 \mathrm{~V}$ of a single Gaussian pulse generator. The pulse peak power has getting to $1.5 \mathrm{~W}$, which is quasi the double of a stand-alone pulse generator.

In order to validate the performance of the circuit proposed, a new compacted design of the powerful monocycle pulse transmitter $U W B$ radar has been implemented in one single-board Fig.13.

To test the efficiency of the designed powerful monocycle pulse transmitter $U W B$ radar sensor, we make some distance measurement form a lossy material which has a high permittivity electric between 30 and 40 . E.g., the $P V C$ Polyvinyl chloride material, a size of $30 \mathrm{~cm} \times 70 \mathrm{~cm} \times 3$ $\mathrm{mm}$ has been chosen to measure the distance accuracy. For this purpose, many measurements for different distance objects ranging between $20 \mathrm{~cm}$ to $60 \mathrm{~cm}$ have been executed. The distance of the target at $60 \mathrm{~cm}$ has been fixed just to confirm the operability of the designed system prototype and their performance in short range application measurements for lossy materials. In order to avoid any electromagnetics perturbation between our system and the environment, the principles of electromagnetic compatibility have been respected, and all measurement has been carrying out in an anechoic chamber. The distance between the trans-
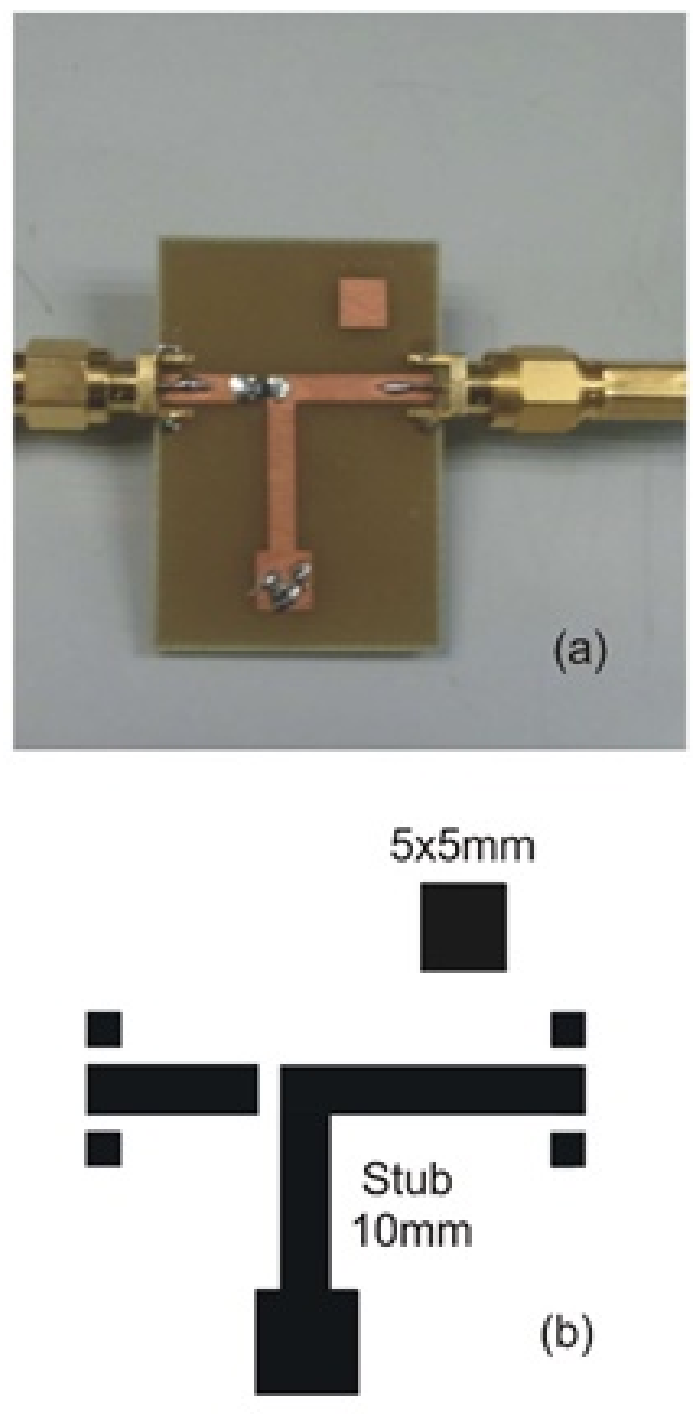

Figure 11: (a) Circuit and (b) Layout of Monocycle forming network.

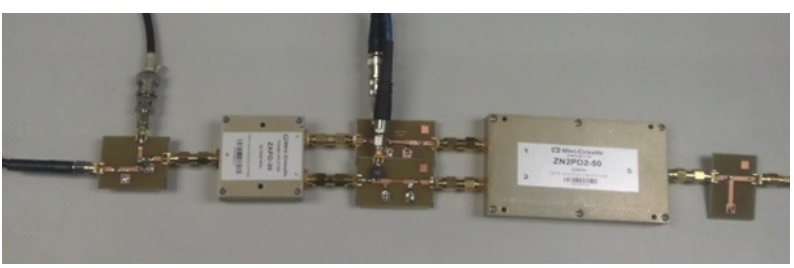

Figure 12: Photo of the ptoposed Radar Transmitter.

mitter $(T x)$ and receiver antenna $(R x)$ has been fixed at 15 $\mathrm{cm}$. The measurement setup of pulse radar sensor for the elected target distance and the photograph of $U W B$ impulse generator are shown in Fig. 14. While Fig.15 and Fig.16 Show the Time-domain response of the radar sensor measurement towards the $P V C$ plate located at $120 \mathrm{~cm}$ and $320 \mathrm{~cm}$ respectively. We observe clearly from the behavior of the curves, the presence of the reference pulse and the reflected pulse from the object accompanied with some unwanted peaks and some ringing in the time-domain re- 

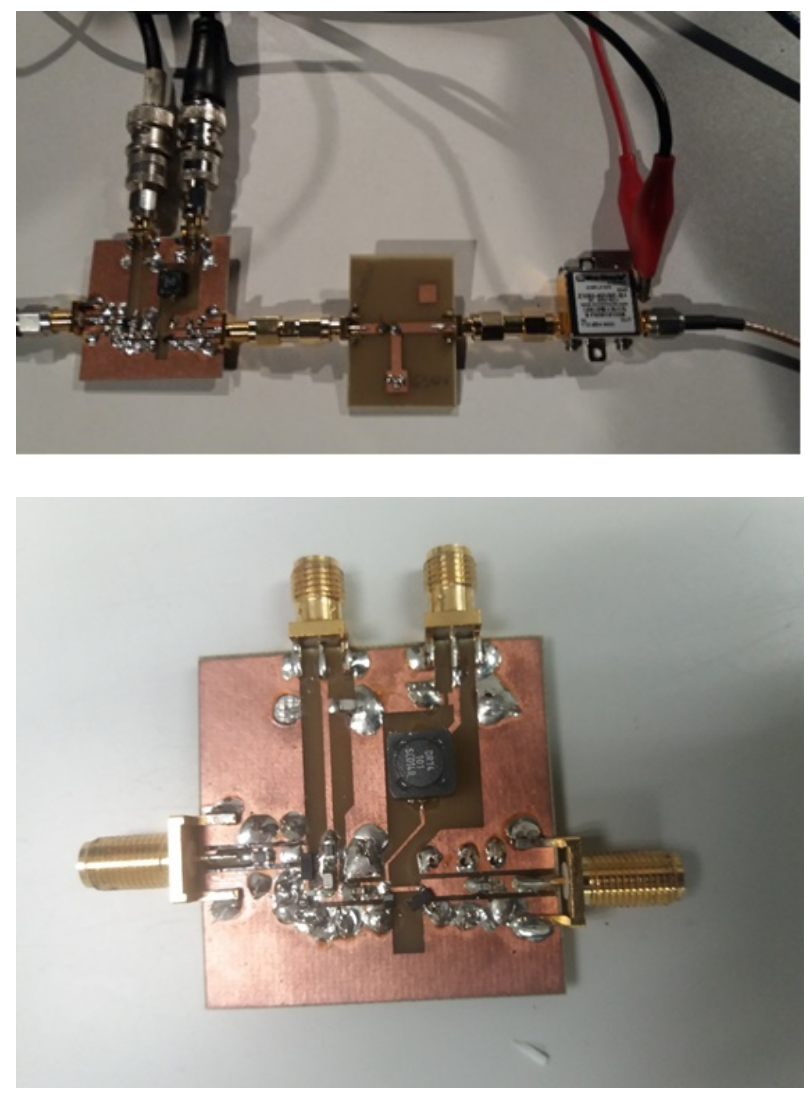

Figure 13: Photo of the proposed radar transmitter circuit.

sponse occurred after the target return is received.

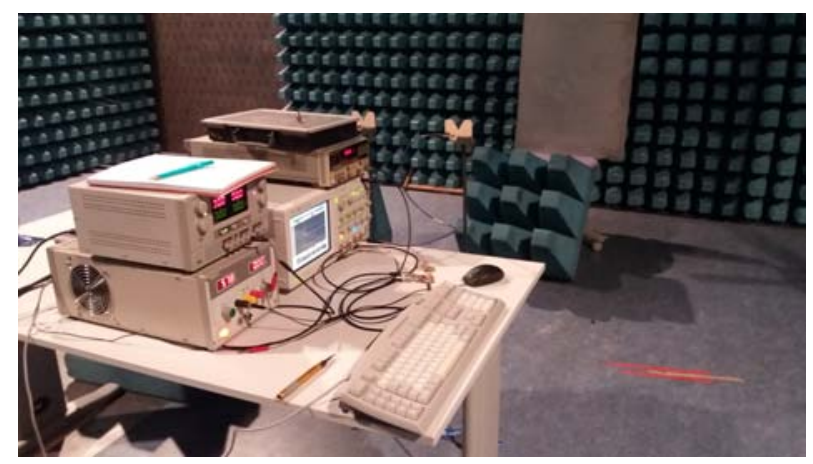

Figure 14: Photograph of measurement setup and Photograph of UWB impulse generator.

More improvement on the signal reception and better use of data at the reception can be taken out with a developed signal processing, but this not the principal aim of this paper. Our purpose in this paper was to report the hardware development and to demonstrate the workability of the radar sensor prototype.

\section{Conclusions}

In This paper, a high power sub-nanosecond pulse transmitter for $U W B$ radar sensor has been presented. The transistor driver and transmission line pulse have been investigated
Distance Measurement for PVC at $1.2 \mathrm{~m}$

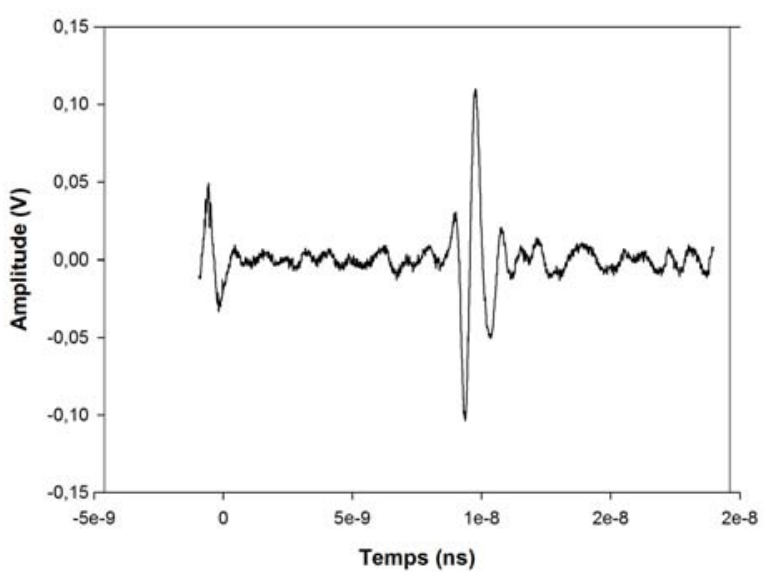

Figure 15: Time-domain response of the radar sensor measurement towards a $P V C$ plate located at $120 \mathrm{~cm}$.

Distance Measurements for PVC at $3.2 \mathrm{~m}$

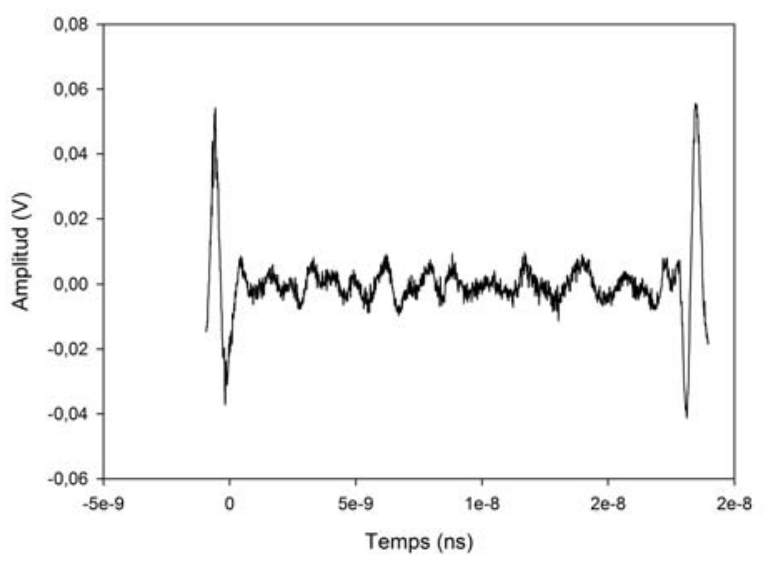

Figure 16: Time-domain response of the radar sensor measurement towards a $P V C$ plate located at $320 \mathrm{~cm}$.

in detail. The principal aim was to transform a square waveform signal to a driving pulse with the timing and the amplitude parameters required by the $S R D$ to form an output Gaussian pulse to generate a powerful monocycle pulses. In order to increase the rise-time of the pulses, a new model of a step recovery diode has been implemented as a sharpener circuit. A simple technique and useful solution have been introduced to increase the output amplitude of the transmitter. This technique consists in assembling two identical generator units on a single board with parallel output connection. The simulation results show that the peak power of summed pulses is nearly double of the peak power of the single one. The pulse transmitter circuit is completely fabricated using micro-strip structure technology characteristics. Waveforms of the generated monocycle pulses over $10 \mathrm{~V}$ in amplitude with $3.5 \%$ in overshoot have been obtained. With an additional output $M F N$, the monocycle pulses have a 9V in amplitude and 600 ps in total width. 
The high agreements between simulated and measurement results show a very high performance of the designed systems. The high power, low cost, and compactness of this monocycle generator make this system very attractive and useful tool for lossy target distance measurements.

\section{Acknowledgement}

The author gratefully acknowledges the financial support provided by Pierre and Marie Curie University under the $E M M A G$ Scholarship. This study was supported by DIMAS group, IT ACA institute at City Polytechnic of innovation from university Polytechnic of Valencia.

\section{References}

[1] Dieter Genschow, Johannes Kloas Evaluation of a UWB radar interface for low power radar sensors, Radar Conference (EuRAD), 2015 European 321324, 2015.

[2] Yu Guo; Guofu Zhu; Zhimin Zhou, A novel design and implementation of sub-nanosecond smpling pulse generator for ultra-wideband equivalent sampling receiver Computing, Communication and Networking Technologies (ICCCNT), 2014 International Conference on page (s): 1-5.

[3] Ahmed abbas Husseien Ameri, Long-Range Ultrawideband Radar Sensor for Industrial Applications Doctoral Diss, Kassel University, November 2012

[4] Ahajjam Younes, Josè M Catala-Civera, Felipe Peñaranda Foix. Abdellah Driouach , Bibliography, Background and overview of UWB radar sensor, Journal of enginieering Research and appliactions, Vol,4, Issue 11 (version-5), november 2014, pp. 37-40.

[5] Amin M. Abbosh, Design of ultra-wideband threeway arbitrary power dividers, IEEE Trans. Microwave Therory Tech vol. 56, no. 1 pp.194- 201, 2008.

[6] E.K. Miller, Time-domain measurements in electromagnetics Springer, New York, 1986.

[7] J.D. Taylor, Ultra-wideband radar technology $C R C$ Press, Boca Raton, FL, 2001.

[8] A. Ouslimani, G. Vernet, H. Hardallah, and R. Adde, Large amplitude picosecond step generation with FETs Electron Lett, 2615631564.

[9] T.-G. Ma, C.-J. Wu, P.K. Cheng, C,-F. Chou, Ultra wideband monocycle pulse generator with dual resistive loaded shunt stubs, IEEE Microw.Opt. Technol vol, pp 459-462.2007.

[10] Z. Li , X. X. Lv , Y. F. Long and T. Qin, An ultra-wideband monocycle pulse generator with good performance Proceedings of Int. Conf. Computat. Problem-Solving (ICCP), pp.253 -254. 2011.
[11] Hewlett-Packard application note AN918, Pulse and waveform generation wih step recovery diodes Hewlett-Packard, Tech. Rep., 1986.

[12] Z. jianming, G, Xiaowei, and F, Yuanchun, A New CAD Model of step Recovery Diode and Generation of UWB signals, IEICE Electronics Expess, Vol, 3, p-p 534-539, Decembre 2006.

[13] Ahajjam Younes, Josè M Catala-Civera, Felipe Peñaranda Foix. Abdellah Driouach, Simple, Compact and low cost Pulse Transmitter for UWB Microwave Radar, Mediterranean Conference on Information \& Technologies Saidia, Morocco, 2015.

[14] Ahajjam Younes, Otman Aghzout, Josè M CatalaCivera, Felipe Peñaranda Foix. Abdellah Driouach , A compact UWB sub-nanosecond pulse generator for microwave radar sensor with ringing miniaturization Multimedia Computing and Systems (ICMCS), 5th International Conference on, Marrakech, Morocco, 2016 .

[15] Amin M. Abbosh, Design of ultra-wideband threeway arbitrary power dividers IEEE Trans. Microwave Therory Tech, vol. 56, no. 1 pp.194-201, 2008.

[16] Han, J., Nguyen, C, A new ultra-wideband, ultrashort monocycle pulse generator with reduced ringing, IEEE Microwave and Wireless Components Letters, vol. 12, no. 6, p. 206-208, 2002. 